\title{
Memory Decline and Depressive Symptoms in a Nationally Representative Sample of Older Adults: The Health and Retirement Study (1998-2004)
}

\author{
Hector M. González ${ }^{a-c}$ Mary E. Bowen ${ }^{a}$ Gwenith G. Fisher ${ }^{c}$ \\ ${ }^{\mathrm{a}}$ Institute of Gerontology and ${ }^{\mathrm{b}}$ Department of Medicine and Public Health Sciences, Wayne State University, \\ Detroit, Mich., and 'Institute of Social Research, University of Michigan, Ann Arbor, Mich., USA
}

\author{
Key Words \\ Depression • Cognitive decline • Older adults • Learning • \\ Memory
}

\begin{abstract}
Background/Aims: Inconsistencies in the relationship between depression and cognitive decline may exist because the expected cognitive domains at risk have not been specified in previous study designs. We aimed to examine the relationship between depressive symptoms and verbal episodic memory functioning over time. Methods: Data from a prospective cohort study (Health and Retirement Study; 1998-2004; $n=18,465$ ), a multistage national probability sample of older adults in the United States, were analyzed. Verbal learning and memory of a 10-word list learning task were the main outcomes. Depressive symptoms (Center for Epidemiologic Studies - Depression Scale) constituted the main predictor. Results: Depressive symptoms were associated with significantly lower immediate $(-0.05 ; p<0.001)$ and delayed $(-0.06 ; p<0.001)$ word list recall scores after controlling for demographics and baseline and time-varying cardiovascular disease risks and diseases. Conclusions: In this US national study of older adults, elevated depressive symptoms were associated with declines in episodic learning and memory over time. These associations were little affected by the demographic or medical conditions consid-
\end{abstract}

\begin{tabular}{ll}
\hline KARGER & ๑ 2008 S. Karger AG, Basel \\
Fax +4161306 1234 & \\
$\begin{array}{l}\text { E-Mail karger@karger.ch } \\
\text { www.karger.com }\end{array}$ & $\begin{array}{l}\text { Accessible online at: } \\
\text { www.karger.com/dem }\end{array}$
\end{tabular}

ered in this study. The results suggest that learning and memory decline may be a long-term feature associated with depressive symptoms among the nation's older adult population.

Copyright $\odot 2008$ S. Karger AG, Basel

\section{Introduction}

Depression is a leading cause of disability in the USA and worldwide [1]. One means by which depression may affect disability is through cognitive dysfunction, which is a symptom of depression [2]. In cross-sectional studies, depression is associated with lower cognitive functioning, particularly episodic memory (also known as declarative memory) [3-6]. In addition, medial temporal lobe brain regions that are associated with episodic memory have been reported to be reduced in people with depression, although not consistently [7]. Further, some reports have indicated improvements in hippocampal activity and cognitive functioning following antidepressant treatment [8], but not in a randomized trial [9]. Depression-related cortisol dysregulation has been suggested as a possible mechanism for the negative changes in brain activity and structure, and episodic learning and memory $[10,11]$.

Several reports from longitudinal studies of the effects of depression have focused on global cognitive function-

\footnotetext{
Hector M. González

Institute of Gerontology, Wayne State University

87 East Ferry Street, 226 Knapp Building

Detroit, MI 48202 (USA)

Tel. +1 313577 2297, Fax +1 313875 0127, E-Mail hmgonzalez@med.wayne.edu
} 
Table 1. Baseline year characteristics (unweighted numbers and weighted percentages) of community-dwelling older adults ( $\geq 51$ years) in the HRS $(1998-2004 ; \mathrm{n}=18,465)$

\begin{tabular}{lcl}
\hline Variables & Respondents & Percent \\
\hline Age groups & & \\
51-59 years & 5,185 & 37.0 \\
60-69 years & 6,342 & 28.4 \\
70-79 years & 4,504 & 23.6 \\
$\quad \geq 80$ years & 2,434 & 11.0 \\
Sex & & \\
$\quad$ Male & 7,598 & 42.8 \\
$\quad$ Female & 10,867 & 57.2 \\
Race & 15,334 & 87.7 \\
$\quad$ White & 3,119 & 12.3 \\
$\quad$ Nonwhite & & \\
Education group & 4,319 & 23.2 \\
0-11 years & 6,278 & 36.3 \\
12 years & 6,869 & 40.5 \\
$\quad \geq 12$ years &
\end{tabular}

ing (e.g., mental status exams), neuropsychological test composite scores or the prediction of dementia and have yielded inconsistent findings [12-14]. In light of work suggesting that depression may selectively affect brain regions associated with verbal episodic learning and memory $[15,16]$, the purpose of this study was to examine the longitudinal association between depressive symptoms and episodic learning and memory in a nationally representative sample of older adults. It was expected that more depressive symptoms over the 6-year study would be associated with lower learning and memory performance.

\section{Methods}

\section{Data}

The Health and Retirement Survey (HRS) is a prospective cohort study that is conducted by the University of Michigan with support from the National Institute of Aging. The first wave of the HRS occurred in 1992 with a 51- to 61-year-old cohort and was merged with the older ( $\geq 70$ years) Asset and Health Dynamics of the Oldest Old Study cohort in 1998. Two additional cohorts were added in 1998 to fill in the gaps between these 2 groups, resulting in a sample design nationally representative of the US population over the age of 50 in 1998. Briefly, the HRS is a multistage probability cohort sample of US households. Further details on the HRS design and methods have been previously published (see Heeringa and Connor [17]).

We analyzed 4 waves of data (1998-2004) from the HRS that were prepared by the RAND Center for the Study of Aging (RAND HRS) for this study. The benefits of using the RAND HRS data include consistent depression and disease reports over the survey period, model-based imputations of missing data and comparability of variables across survey waves (see RAND HRS [18] for details). We analyzed data from 18,465 community-dwelling respondents who were $\geq 51$ years in 1998 . The HRS sample demographic characteristics are presented in table 1. Respondents with sampling weights of 0 indicating they were institutionalized or deceased were excluded from this study. Data were weighted using HRS respondent level sampling weights to account for the sample design in the HRS (Heeringa and Connor [17]). After 4 waves (1998-2004), the combined year response rate was $72.3 \%$. The wave-to-wave reinterview response rates ranged from $92.1 \%$ (2000) to $87.5 \%$ (2004).

\section{Measures}

The main variables of interest in this study were administered at every wave (i.e., every 2 years) of the HRS (1998-2004) and were time-varying. The outcome variables of interest in this study were the immediate and delayed memory scores of a 10-word list learning task administered to all capable HRS respondents. In telephone and face-to-face interviews, 3 alternative lists of 10 common nouns were read to respondents at a rate of 1 per second. Immediately after the word list had been read, the respondents were asked to repeat as many words as they could recall in any order (immediate). After about 3 min of interference tasks, the respondents were asked to recall the 10-word list again (delayed). The scores ranged from 0 to 10 words recalled for the immediate and delayed trials. The interview modes (i.e., telephone and faceto-face) and the use of 4 alternative list forms have been found to have minimal effects on performance; however, learning effects from previous test exposures were reported [19].

The main predictor variable of interest was depression, measured by a Center for Epidemiologic Studies - Depression Scale (CES-D) [20]. The CES-D is a self-reported inventory of depressive symptoms ('was depressed', 'everything was an effort', 'sleep was restless', 'was happy', 'felt lonely', 'enjoyed life', 'felt sad' and 'could not get going') that occurred in the week prior to the respondents' interview date. The HRS uses a version of 8 items of the full 20-item CES-D which were selected based on factor analyses [21]. The 8-item CES-D version reportedly has similar symptom dimensions as the original CES-D and high internal consistency and validity in the HRS $[22,23]$. In this study, the baseline internal consistency reliability coefficient $(\alpha)$ of the CES-D scale was 0.77 . The dichotomously scored CES-D items ranged from 0 to 8 , with 8 indicating the most depressive symptoms in this study. Most of the respondents in this community-dwelling sample reported few depressive symptoms overall (baseline mode $=0$, mean $=1.62$; see Wallace et al. [23]). Additionally, demographic variables (non-time-varying) and time-varying self-reported risk factors for vascular disease or vascular disease were included as covariates. The demographic measures included: sex (male $=0$, female $=1)$, race (white $=0$, nonwhite $=1)$, education $(0-17$ or more years) and age in years. Age was centered at 65 years for ease of interpretation and to restrict problems with collinearity [24, 25]. Time-varying diabetes, hypertension, stroke and cardiovascular disease (CVD) were coded dichotomously as present or absent. Diabetes and hypertension were aggregated to indicate CVD risk; stroke and CVD were aggregated to indicate CVD. At baseline, the respondents were asked if a doctor had ever told them that they had these conditions (i.e., measure of prevalent conditions). In later survey waves, the respondents were asked if they 
had been told by a doctor (since the prior interview) that they had developed any of these conditions (i.e., measure of incident conditions). Reasonable concordance values between self-reports of disease and medical chart reviews have been reported.

\section{Analytic Approach}

Multilevel statistical modeling using Hierarchical Linear Modeling software version 6.02 (Scientific Software International, Lincolnwood, Ill., USA) was used to examine individual and aggregate levels of data over time [24]. Basically, multilevel modeling in Hierarchical Linear Modeling conceives of each individual as having their own regression equation. We present our hierarchical models as a series of nested models, 1 for each level of the hierarchy. At the first level, each individual respondent's trajectory of change in learning and memory was represented as a function of person-specific parameters (e.g., risk for CVD, CVD, CESD) plus random error. The second level statistically modeled individual variations in growth parameters across a population of persons (e.g., sex, race, education and the number of previous word list exposures). The third level additionally adjusted our analyses for same household respondents, as cohabitating individuals in the same household may be more similar to one another than households with only 1 respondent. The multilevel assumption of normality was examined and revealed no violation of this assumption.

Two separate pairs of multilevel models were used to examine immediate and delayed word list scores. In both models, the count of depressive symptoms endorsed was the main predictor, with covariates for sex, race, education, word list exposure frequency and households with $>1$ respondent (see below).

\section{Results}

\section{Bivariate Results}

Table 1 presents the baseline characteristics of the sample. As shown in table 1, the sample was largely white and female. Most respondents were between the ages of 60 and 69 years $($ mean $=66.9)$ at the 1998 baseline year and most respondents had $\geq 12$ years of education. Table 2 displays the weighted means and standard deviations of the immediate and delayed word list scores by interview regardless of wave. As table 2 shows, the respondent's average immediate and delayed word list scores decreased from their first interview to their third interview and increased in their fourth interview. The immediate and delayed recall score increases in the fourth interview may have been associated with learning effects.

The incidence of acute and chronic conditions increased with each survey wave (not shown). At baseline, about $20 \%$ of the respondents reported ever having risks for CVD, while $21 \%$ of the respondents reported ever having CVD. In 2004, the 6-year incidence of new self-reported cases of CVD risks was $1.5 \%$ and $8 \%$ for CVD.
Table 2. Weighted learning (immediate) and memory (delayed) score averages from a 10-word list learning task by respondent interview in the HRS $(1998-2004 ; \mathrm{n}=18,465)$

\begin{tabular}{|c|c|c|c|c|c|}
\hline & \multicolumn{2}{|c|}{$\begin{array}{l}\text { Immediate word } \\
\text { list recall }\end{array}$} & \multicolumn{2}{|c|}{$\begin{array}{l}\text { Delayed word } \\
\text { list recall }\end{array}$} & \multirow[b]{2}{*}{ Subjects } \\
\hline & M & SD & M & SD & \\
\hline \multicolumn{6}{|c|}{ Respondent interview } \\
\hline 1 st & 5.74 & 1.82 & 4.68 & 2.18 & 18,465 \\
\hline 2nd & 5.30 & 1.84 & 4.17 & 2.11 & 17,015 \\
\hline $3 \mathrm{rd}$ & 5.16 & 1.81 & 4.12 & 2.12 & 15,268 \\
\hline 4 th & 5.44 & 1.68 & 4.39 & 2.03 & 13,358 \\
\hline
\end{tabular}

Scores are calculated based on the respondent's interview regardless of the wave year. For example, interview 1 indicates the first interview the respondent had, which could be on or after wave year 1998. $\mathrm{M}=$ Mean; $\mathrm{SD}=$ standard deviation.

\section{Multivariate Results}

Immediate Word List Results. Table 3 (model 1) shows the relationship between CES-D and immediate word list recall scores after adjustment for demographic variables.

In model 1, CES-D was negatively associated with immediate word list scores $(-0.06 ; \mathrm{p}<0.001)$. That is, with each depressive symptom, the respondents recalled 0.06 fewer words. Model 2 was additionally adjusted for baseline and time-varying CVD risk factors and CVD. The variables added to model 2 did not significantly affect the association between CES-D and immediate word list recall scores. That is, with each depressive symptom, the respondents continued to recall fewer words $(-0.05 ; \mathrm{p}<$ 0.001). Risks for CVD ( $p<0.001)$ and CVD ( $p<0.001)$ were associated with fewer words recalled, and the average respondent with CVD recalled fewer words $(-0.14$; $\mathrm{p}<0.001)$ than the average respondent with CVD risks $(-0.07 ; \mathrm{p}<0.001)$. A hypothesis test was used to compare immediate models 1 and 2 by examining the significance of the change in $-2 \log$ likelihood $\chi^{2}$ between the models, the results of which are shown at the bottom of table 3 . Though adjustments for CVD risk and CVD did not better explain the relationship between CES-D and immediate word list scores, the model-fit statistics show that these adjustments in the immediate model 2 did improve the model fit to the data, with a significant $(\mathrm{p}<0.001)$ change in the $-2 \log$ likelihood $\chi^{2}(288.81$; d.f. $=2)$.

Delayed Word List Recall Results. Table 3 shows the relationship between CES-D and delayed word list recall scores after demographic adjustments. In model 1, 
Table 3. Estimated associations between learning and memory (immediate and delayed word list recall scores) and depressive symptoms (CES-D scale) in US community-dwelling adults (ages $\geq 51$ years) from the HRS (1998-2004)

\begin{tabular}{|c|c|c|c|c|c|c|c|c|}
\hline & \multicolumn{4}{|c|}{ Immediate word list } & \multicolumn{4}{|c|}{ Delayed word list } \\
\hline & \multicolumn{2}{|l|}{ Model 1} & \multicolumn{2}{|l|}{ Model 2} & \multicolumn{2}{|l|}{ Model 1} & \multicolumn{2}{|l|}{ Model 2} \\
\hline & Coefficients & SE & Coefficients & SE & Coefficients & SE & Coefficients & SE \\
\hline Mean word list score & $3.05^{*}$ & 0.04 & $3.16^{*}$ & 0.05 & $1.69^{*}$ & 0.05 & $1.83^{*}$ & 0.06 \\
\hline Mean word list growth rate & $-0.07^{*}$ & 0.00 & $-0.07^{*}$ & 0.00 & $-0.08^{*}$ & 0.00 & $-0.08^{*}$ & 0.00 \\
\hline Depressive symptoms & $-0.06^{*}$ & 0.00 & $-0.05^{*}$ & 0.00 & $-0.07^{*}$ & 0.00 & $-0.06^{*}$ & 0.00 \\
\hline CVD risk factors & & & $-0.07^{*}$ & 0.01 & & & $-0.10^{*}$ & 0.01 \\
\hline CVD events & & & $-0.14^{*}$ & 0.02 & & & $-0.16^{*}$ & 0.01 \\
\hline \multicolumn{9}{|l|}{ Model fit } \\
\hline Deviance & $215,817.28$ & & $215,528.47$ & & $234,944.55$ & & $234,687.73$ & \\
\hline Estimated parameters & 10 & & 12 & & 10 & & 12 & \\
\hline \multicolumn{9}{|l|}{ Model comparisons } \\
\hline Model 1 vs. model 2 & \multirow{2}{*}{\multicolumn{4}{|c|}{$\Delta \mathrm{x}^{2}=288.81 ;$ d.f. $=2 ; \mathrm{p}<0.001$}} & \multirow{2}{*}{\multicolumn{4}{|c|}{$\Delta \mathrm{x}^{2}=246.82 ;$ d.f. $=2 ; \mathrm{p}<0.001$}} \\
\hline Model 3 vs. model 4 & & & & & & & & \\
\hline
\end{tabular}

Models are adjusted for immediate and delayed word list exposure frequency and married/partnered couples. Based on responses to a 10 -word immediate and delayed recall word list. ${ }^{*} \mathrm{p}<0.001$.

CES-D was negatively associated with delayed word list recall $(-0.07 ; \mathrm{p}<0.001)$. That is, with each depressive symptom, there were 0.07 fewer words recalled in the delayed word list trial. The relationship between delayed word list recall scores and CES-D remained largely unchanged after controlling for CVD risk factors and CVD in the delayed model $2(-0.06 ; \mathrm{p}<0.001)$. That is, in the delayed model 2 , with each additional depressive symptom, word recall decreased by $0.06(\mathrm{p}<0.001)$. CVD risk and CVD were associated with fewer words recalled.

The average respondent with CVD conditions recalled fewer words $(-0.16 ; \mathrm{p}<0.001)$ than the average respondent who was just at risk for CVD $(-0.10 ; \mathrm{p}<0.001)$. A hypothesis test was used to examine the significance of the change in $-2 \log$ likelihood $\chi^{2}$ between the delayed word list recall models 1 and 2, the results of which are shown at the bottom of table 2. Though CVD risk and CVD did not better explain the relationship between delayed word recall and CES-D, model-fit statistics show that these adjustments in the delayed model 2 did improve the model fit to the data, with a significant $(\mathrm{p}<$ $0.001)$ change in the $-2 \log$ likelihood $\chi^{2}(246.82$; d.f. $=2)$. In sum, as CES-D increased, the number of words recalled in both the immediate and delayed word recall trials decreased. The relationship between CES-D and immediate and delayed word list recall was not better explained by demographics or baseline and time-varying adjustments for CVD risk factors and CVD.

\section{Conclusion}

Over the course of 6 years, elevations in depressive symptoms were associated with lower verbal episodic learning and memory scores in this nationally representative sample of community-dwelling older adults. A major finding of this study was that by specifying a cognitive domain that is associated with chronic stress and the negative affects of depression in both nonhuman and human studies $[11,26]$, we were able to demonstrate verbal episodic learning and memory decline over time. These findings suggest that depressive conditions that affect many older adults may be related to dysfunction of brain regions associated with verbal episodic learning and memory. This is particularly important since memory loss is the vanguard of dementia in most patients [2]. The findings presented here support the hypothesis that chronic stress and depression are associated with failing hippocampal function [10]. Clinically, these findings support the regular practice of depression screening of patients with memory complaints [14].

Previous longitudinal studies of depression have not consistently found cognitive decline. Most of these studies have not specified the cognitive domains that would be expected to be negatively affected by depression, which may explain inconsistencies in the findings of earlier work. For example, several studies have relied on global cognitive function measures, such as the Mini Mental 
State exam, or neuropsychological batteries reduced to composite scores for identifying cognitive impairment or dementia [12-14, 27]. Findings from the longitudinal studies that provided verbal episodic learning and memory performance information are mixed, which may be related to attrition, the limited availability of follow-up waves of information and the methods used for longitudinal data analyses [14, 28].

The results presented here are consistent with crosssectional findings of associations between depressive symptoms and lower verbal episodic learning and memory performance $[3,15]$. In these cross-sectional and many of the longitudinal studies above, the neurotoxic effect of cortisol dysregulation on hippocampal function was considered as a possible explanation for the lower verbal episodic learning and memory performance and hippocampal atrophy on MRI for depressed participants $[15,16]$. The verbal episodic learning and memory decline results presented here are supportive of the hypothesis that depressive symptom related allostatic load negatively affected hippocampal functioning in this sample.

A major strength of this study is that it used multilevel statistical analyses that considered within- and between-person estimates, and time-varying depression, medical conditions and other nonvarying covariates. Multilevel analyses account for autocorrelation problems associated with repeated measures analyses that may have affected analyses in previous reports. Few of the previous reports examining the association between depression and cognitive change have accounted for these important factors in their analytic approaches. The studies that have analyzed their data using similar approaches to this study have found associations between depressive symptoms and cognitive decline $[13,14]$. To the best of our knowledge, the results presented here from HRS represent the largest study of the association between depression and cognitive decline. Because our results represent national estimates, they can be generalized to the entire population of noninstitutionalized, communitydwelling older adults in the USA.

The results of this study indicate that depressive symptoms are associated with verbal episodic learning and memory decline. The significant declines were not explained by other important medical comorbidities and demographic information. Indeed, risk factors for vascular disease and vascular diseases themselves were independently associated with verbal learning and memory decline. Although the focus of this report was not on the medical conditions used in this study, the results present- ed here are consistent with previous work indicating associations between vascular disease and cognitive change $[29,30]$. Nevertheless, it remains possible that other unaccounted variables may explain the verbal episodic learning and memory decline associated with depressive symptoms found in this sample, and there are several limitations to consider when interpreting the results of this study. Although we were able to capture time-varying depressive symptoms over the 6-year period of this study, we were not able to determine the level of depressive symptoms respondents may have experienced outside of the time periods examined. Secondly, other attentional and motivational factors not examined in this study may have contributed to the negative association between depressive symptoms and episodic learning and memory that were reported herein. Additionally, the response rate over the study period was high (72.3\%). Nevertheless, attrition remains an important concern. Over the 6-year period of this study, the response rate of HRS respondents with elevated depressive symptoms (i.e., CES-D $\geq 4$ ) was lower $(68.5 \%)$ than that of respondents with fewer symptoms. The bias introduced by selective, depression-related attrition was likely to have been small; however, any selective, depression-related attrition would likely have resulted in underestimates of the association between depressive symptoms and cognitive decline.

The relationship between depressive symptoms and verbal episodic learning and memory over the 6 years of this study was small, but the rate of decline is similar to that reported by Wilson et al. [14]. The following is an example of how the results of this study might translate at the individual level: a 70 -year-old white woman with 12 years of education, diabetes and an endorsement of all 8 depressive symptoms would recall an estimated 4 words (4.06) on the delayed memory trial at baseline. With CESD scores of 8 consistent across years, her estimated scores would drop to about half of a point ( 3.47 words) after 6 years and below 3 (2.87 words) after 12 years (about the 10 th percentile). This example of depressive symptom related verbal episodic memory decline is small and the threshold points of concern from the individual or significant others are not certain. However, this example of memory loss approaches clinical significance and may pose functional problems for the individual and family. The average level of depressive symptoms in this community-dwelling population was small. In populations with higher levels of depressive symptoms (e.g., older Mexican Americans) or among patients with chronic clinical depression, the effect on episodic learning and memory may be higher [31]. For many older adults, de- 
pressive symptoms are accompanied with a vascular disease event, such as myocardial infarction, which would likely contribute to further memory loss and impairment. The association between depressive symptoms and cognitive decline supports the practice of depression screening for older Americans, particularly those with cognitive complaints.

\section{Acknowledgments}

Dr. González receives funding from the National Institute of Mental Health (MH 67726). Dr. Bowen is supported by a postdoctoral training grant awarded to the Wayne State University, Institute of Gerontology, by the Agency for Healthcare Research and Quality. The authors wish to express their gratitude to Dr. David Steffens for reviewing the manuscript and providing insightful comments.

\section{References}

-1 McKenna MT, Michaud CM, Murray CJ, Marks JS: Assessing the burden of disease in the United States using disability-adjusted life years. Am J Prev Med 2005;28:415-423.

2 APA: Diagnostic and Statistical Manual of Mental Disorders, ed 4, rev. Washington, American Psychiatric Association, 2000.

3 Airaksinen E, Larsson M, Lundberg I, Forsell Y: Cognitive functions in depressive disorders: evidence from a population-based study. Psychol Med 2004;34:83-91.

-4 Ilsley JE, Moffoot AP, O’Carroll RE: An analysis of memory dysfunction in major depression. J Affect Disord 1995;35:1-9.

5 Kindermann SS, Brown GG: Depression and memory in the elderly: a meta-analysis. J Clin Exp Neuropsychol 1997;19:625-642.

6 King DA, Caine ED (eds): Neuropsychological Assessment of Neuropsychiatric Disorders, ed 2. New York, Oxford University Press, 1996.

7 Von Gunten A, Fox NC, Cipolotti L, Ron MA: A volumetric study of hippocampus and amygdala in depressed patients with subjective memory problems. J Neuropsychiatry Clin Neurosci 2000;12:493-498.

8 Mayberg HS, Brannan SK, Tekell JL, Silva JA, Mahurin RK, McGinnis S, et al: Regional metabolic effects of fluoxetine in major depression: serial changes and relationship to clinical response. Biol Psychiatry 2000;48: 830-843.

9 Nebes RD, Pollock BG, Houck PR, Butters MA, Mulsant BH, Zmuda MD, et al: Persistence of cognitive impairment in geriatric patients following antidepressant treatment: a randomized, double-blind clinical trial with nortriptyline and paroxetine. J Psychiatr Res 2003;37:99-108.

10 Lee BK, Glass TA, McAtee MJ, Wand GS, Bandeen-Roche K, Bolla KI, et al: Associations of salivary cortisol with cognitive function in the Baltimore Memory Study. Arch Gen Psychiatry 2007;64:810-818.

11 Sapolsky RM: The possibility of neurotoxicity in the hippocampus in major depression: a primer on neuron death. Biol Psychiatry 2000;48:755-765.
Cervilla JA, Prince M, Joels S, Mann A: Does depression predict cognitive outcome 9 to 12 years later? Evidence from a prospective study of elderly hypertensives. Psychol Med 2000;30:1017-1023.

13 Chodosh J, Kado DM, Seeman TE, Karlamangla AS: Depressive symptoms as a predictor of cognitive decline: MacArthur Studies of Successful Aging. Am J Geriatr Psychiatry 2007;15:406-415.

14 Wilson RS, Mendes De Leon CF, Bennett DA, Bienias JL, Evans DA: Depressive symptoms and cognitive decline in a community population of older persons. J Neurol, Neurosurg Psychiatry 2004;75:126-129.

15 Sheline YI, Sanghavi M, Mintun MA, Gado $\mathrm{MH}$ : Depression duration but not age predicts hippocampal volume loss in medically healthy women with recurrent major depression. J Neurosci 1999;19:5034-5043.

16 Mintun MA, Sheline YI, Moerlein SM, Vlassenko AG, Huang Y, Snyder AZ: Decreased hippocampal 5-HT2A receptor binding in major depressive disorder: in vivo measurement with $[18 \mathrm{~F}]$ altanserin positron emission tomography. Biol Psychiatry 2004;55:217224.

17 Heeringa SG, Connor J: Technical Description of the Health and Retirement Study Sample Design. HRS/AHEAD Documentation Report DR-002. Ann Arbor, Survey Research Center at the Institute for Social Research, 1995.

18 RAND HRS Data, Version F. National Institute on Aging and Social Security Administration, 2006.

19 Rodgers WL, Ofstedal MB, Herzog AR: Trends in scores on tests of cognitive ability in the elderly US population, 1993-2000. J Gerontol B Psychol Sci Soc Sci 2003;58: S338-S346.

20 Radloff L: The CES-D Scale: a self-report depression scale for research in the general population. Appl Psychol Meas 1977;1:385401.

21 Radloff LS, Teri L: Use of the Center for Epidemiological Studies-Depression Scale with older adults. Clin Gerontol 1986;5:119-136.
22 Steffick DE: Documentation of Affective Functioning Measures in the Health and Retirement Study. HRS Documentation Report DR-005. Ann Arbor, Survey Research Center at the Institute for Social Research, 2000.

23 Wallace R, Herzog AR, Ofstedal MB, Steffick D, Fonda S, Langa K: Documentation of Affective Functioning Measures in the Health and Retirement Study. Ann Arbor, University of Michigan, 2000.

24 Raudenbush S, Bryk AS: Hierarchical Linear Models, Applications and Data Analysis Methods. Thousand Oaks, Sage Publications, 2002.

25 West BT, Welch KB, Galecki AT: Linear Mixed Models. A Practical Guide Using Statistical Software. Boca Raton, Chapman Hall/CRC Press, 2007.

26 Sapolsky RM: Glucocorticoids, stress, and their adverse neurological effects: relevance to aging. Exp Gerontol 1999;34:721-732.

27 Chen P, Ganguli M, Mulsant BH, DeKosky ST: The temporal relationship between depressive symptoms and dementia: a community-based prospective study. Arch Gen Psychiatry 1999;56:261-266.

28 Comijs HC, Jonker C, Beekman AT, Deeg DJ: The association between depressive symptoms and cognitive decline in communitydwelling elderly persons. Int J Geriatr Psychiatry 2001;16:361-367.

29 Elias MF, Elias PK, Sullivan LM, Wolf PA, D’Agostino RB: Obesity, diabetes and cognitive deficit: The Framingham Heart Study. Neurobiol Aging 2005;26(suppl 1):11-16.

30 Wolf PA, Beiser A, Elias MF, Au R, Vasan RS, Seshadri S: Relation of obesity to cognitive function: importance of central obesity and synergistic influence of concomitant hypertension: The Framingham Heart Study. Curr Alzheimer Res 2007;4:111-116.

31 González HM, Haan MN, Hinton L: Acculturation and the prevalence of depression in older Mexican Americans: baseline results of the Sacramento Area Latino Study on Aging. J Am Geriatr Soc 2001;49:948-953. 